\title{
Comparative Analysis of Machine Learning Techniques for the Classification of Knee Abnormality
}

\author{
$1^{\text {st }}$ Ankit Vijayvargiya \\ Department of Electrical Engineering \\ $2^{\text {nd }}$ Rajesh Kumar \\ $3^{\text {rd }}$ Nilanjan Dey \\ Malviya National Institute of Technology Malviya National Institute of Technology \\ Jaipur, India \\ ankitvijayvargiya29@gmail.com \\ Jaipur, India \\ rkumar.ee@mnit.ac.in \\ Department of Information Technology \\ Techno India College of Technology \\ Kolkata, India \\ neelanjan.dey@gmail.com
}

\author{
$4^{\text {th }}$ João Manuel R. S. Tavares \\ Instituto de Ciência e Inovação em Engenharia Mecânica e Engenharia Industrial \\ Departamento de Engenharia Mecânica \\ Faculdade de Engenharia, Universidade do Porto \\ Porto, Portugal \\ tavares@fe.up.pt
}

\begin{abstract}
Knee abnormality is a major problem in elderly people these days. This can be diagnosed by using MRI or $X$-Ray techniques. X-Ray technique is only be used for the primary evaluation while MRI is an efficient way to diagnose knee abnormality but it is very expensive. In this work, Surface EMG (sEMG) signal taken from healthy and knee abnormal individuals for three different lower limb movements (Gait, Standing and Sitting) were used for the classification. For the classification, first Discrete Wavelet Transform (DWT) was being used for denoising the signal then eleven different time-domain features were extracted by using 256 msec windowing with $25 \%$ overlapping. After that normalized the features between 0 to 1 and then selected by using the backward elimination method using the pvalue test. Five different machine learning classifiers (k-nearest neighbor, support vector machine, decision tree, random forest, and extra tree) were tested for this classification by training with selected features. Our result shows that the Extra Tree Classifier with ten cross-validations gives the highest accuracy (91\%) to detect the knee abnormality using sEMG signal.

Index Terms-Knee Abnormality, Surface Electromyography (sEMG), Discrete Wavelet Transform (DWT), Machine Learning Classifiers.
\end{abstract}

\section{INTRODUCTION}

In the present scenario, knee pain is a most common healthcare issue in the elderly. The major reasons behind the knee pain are related to injury, aging, repeated stress on the knee or due to an underlying condition such as knee arthritis. According to the united-state study, more than one in ten adults suffer from knee osteoarthritis (Knee OA) which is a form of knee abnormality [1].

The Knee joint is one of the complex joints in the human body which provides the leg movement, the stability of the human body, and also acts as a shock absorber. Bones, ligaments, tendons, muscles, cartilage, and fluids are the different parts of the knee joint. Tibia, femur, and pattela are the three major bones that are used to form the knee joint.
X-Ray, Magnetic Resonance Imaging (MRI), CT, Arthroscopic are the different techniques that are being used to diagnose the knee abnormality clinically [2], [3], [4]. XRay is being used for the initial evaluation of knee pain but this technique is not powerful. Clinically, magnetic resonance imaging (MRI) procedure is commonly used to assess knee pain but is very costly. As per the literature, knee abnormality can also be diagnosed with the help of human activities by using wearable sensor like EMG, Gyrometer, Accelerometer [5], [6], [7] and visual sensor like camera [8], [9]. Privacy, pervasiveness, and complexity are some of the advantages of wearable sensors over the visual sensors. In the wearable sensors, EMG sensors are better in the human activity recognition applications because it can recognize the movement in advance and also take less time to detect the signal.

Electromyography (EMG) is a technique used to analyze and record the electrical activity emitted during muscle contraction by the skeletal muscles. Surface EMG (sEMG) and intramuscular EMG (iEMG) are the two ways by which collect the EMG signal [10]. Recording of sEMG signal has several advantages over iEMG signals. The sEMG signals play a critical role in analyzing the lower limb movements and may help in detecting anomalies in the lower limb. Artificial Neural Network based knee abnormality classification has been done by Erkamaz et al [4]. Vijayvargiya et al have been analyzed the early detection of knee osteoarthritis by using support vector machine classifier with different kernels [11]. Ertugrul et al. have been used surface EMG signal classification of the lower limb and upper limb by using adaptive local binary patterns [12].

In this work, we present a comparative analysis of the performance of various machine-learning classifiers for knee 
Walking

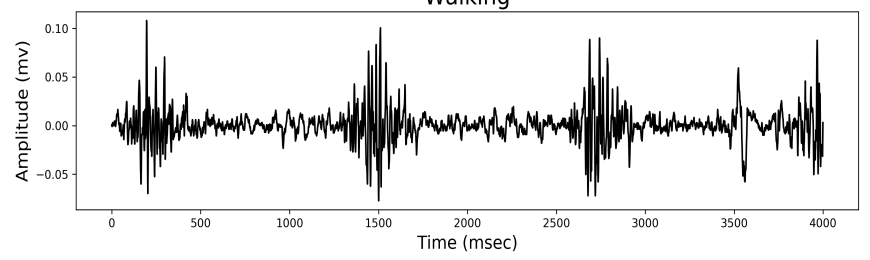

Sitting

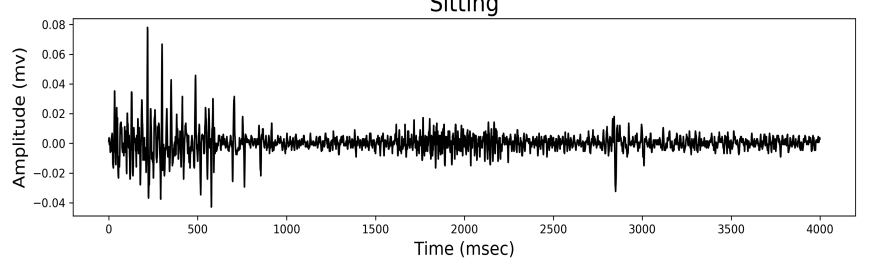

Standing

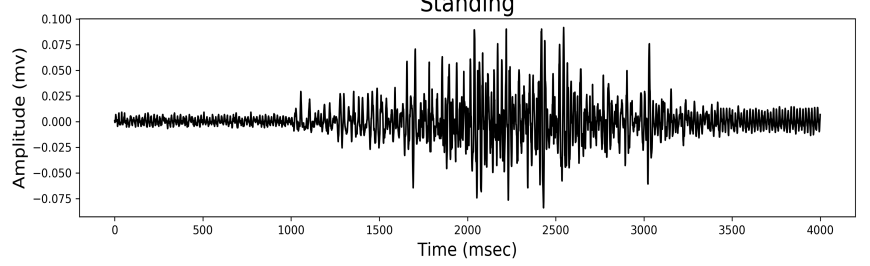

(a)

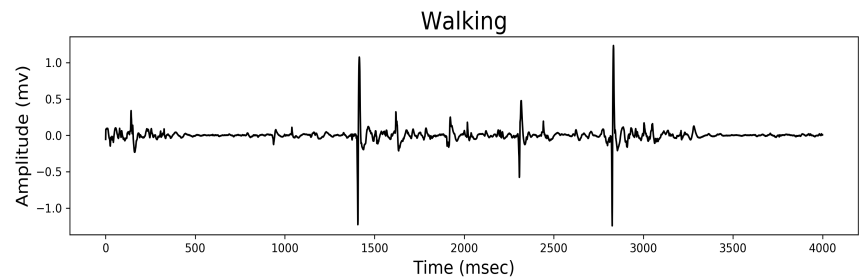

Sitting

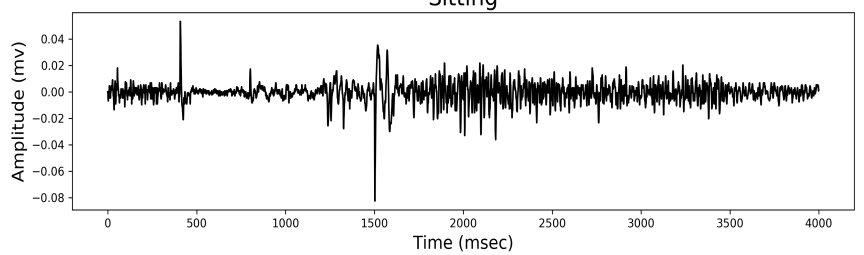

Standing

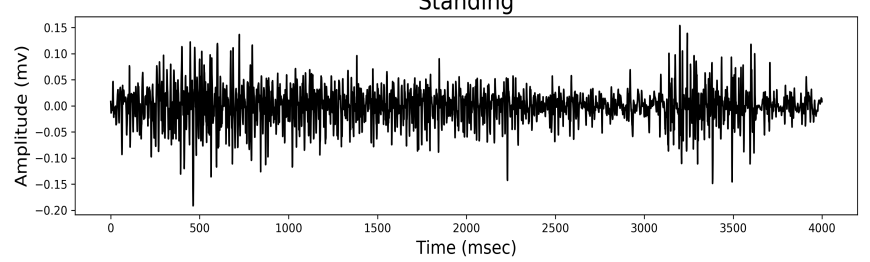

(b)

Fig. 1: sEMG signal taken for three different postures (Walking, Standing, Sitting) : a) Normal subject b) Abnormal subject

abnormality detection using sEMG datasets. This paper is divided into five sections. A brief overview of the EMG data set is provided in Section II. The detail of the proposed methodology is shown in section III. Results and discussion are presented in section IV. Conclusions and possible research are discussed in section $\mathrm{V}$.

\section{DATASET}

In this paper, we were considered the datasets which consist of the surface EMG signal from three different movements (standing, walking and sitting) of twenty-two subjects [13]. The age of all the subjects were more than 18 years in 11 individuals were healthy and 11 individuals were suffering from knee abnormalities. The healthy individuals did not have any record of a knee injury while the unhealthy individuals have suffering knee abnormality which was already diagnosed by professionals. DataLog MWX8 and a goniometer were used to collect the surface EMG data for three different postures. The data were collected around the four distinct muscles: rectus femoris (RF), biceps femoris (BF), vastus medialis (VM), and semitendinosus (ST). The goniometer was attached to the external side of the knee joint. All these data were stored on the computer by the bluetooth adapter. For this analysis, we have considered only EMG signals for three distinct movements. Fig 1 shows an example of the normal and abnormal subject's sEMG recordings in each posture, respectively.

\section{Proposed Methodology}

This section gives a brief description of the methodology used in knee abnormality detection by using surface EMG datasets. Fig. 2 illustrates the basic steps involved in this classification of the sEMG signal. First, the discrete wavelet transform (DWT) was used to denoised the raw sEMG signal and then various features were extracted using overlapping windowing techniques. To reduce the feature dimension and improve performance parameters, a backward elimination method for feature selection was used. After that, selected features were fed to the machine learning classifiers and analyzed the performance parameters.

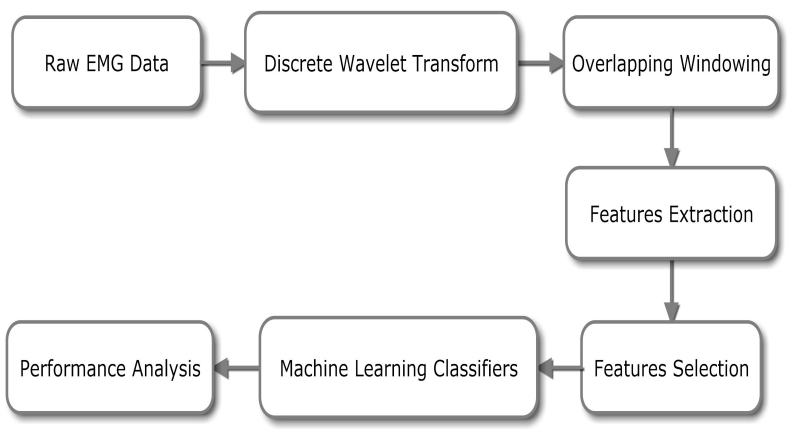

Fig. 2: Basic flow chart of the proposed methodology

\section{A. Discrete Wavelet Transform}

During the recording of the sEMG signal, Several different kinds of noises are introduced in the data. The noises are present due to external disturbances and psychological disturbances. Owing to the combination of various noise signals or artifacts, the identity of an EMG signal gets lost. 
The EMG signal attributes depend on the skin temperatures, subject's internal structure, skin formation, blood flow rate, tissue structure, measurement location, and more.

The identity of an EMG signal is lost due to the mixing of various noise signals or artifacts. The attributes of the EMG signal depend on the internal structure of the subject, including the individual skin formation, blood flow velocity, measured skin temperatures, the tissue structure (muscle, fat, etc.), the measuring site, and more.

It is not possible to use traditional filtering techniques such as high-pass, low-pass, and band-pass to eliminate unwanted noise like impulse within the active EMG signal spectrum band. Novel techniques including Independent Component Analysis (ICA), Discrete Wavelet Transform (DWT), and Empirical Mode Decomposition (EMD) have recently been used to eliminate random noise from sEMG signals. [14], [15], [16], [17], [18]. In this work, DWT was applied to denoise the raw sEMG signal because it has minimum signal distortion and gives information in both frequency and time domain.

The signal can decomposed into various levels in a discrete wavelet transformation using various wavelets such as Haar, Daubechies, Marlet, Symlet, etc. It is implemented as a bank of filters which contains low pass filters (approximate coefficient) and high pass filters (detail coefficient). Further, the signal is passed through the next level of LPF and HPF. The number of coefficients depends on the level of decomposition. Fig. 3 shows the wavelet decomposition up to level 4 . The wavelet are generated from a mother wavelet $\left(\psi_{t}\right)$, by scaling (s) and translation $(\tau)$ [19]:

$$
\psi_{s, \tau}=\frac{1}{\sqrt{s}} \psi\left(\frac{t-\tau}{s}\right)
$$

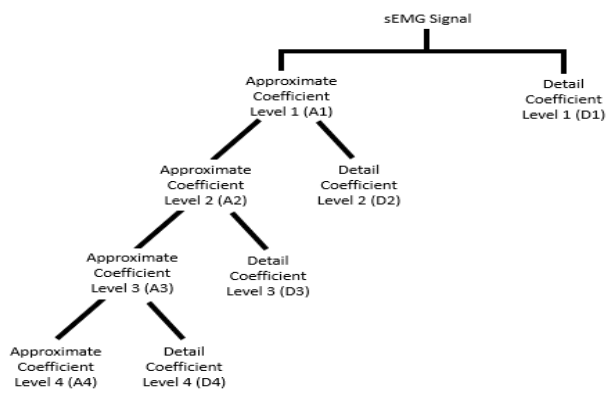

Fig. 3: Wavelet Decomposition

To remove the noise after decomposing the signal, various kind of thresholding such as soft thresholding, hard thresholding have been used. In this study, wavelet denoising was used with $\mathrm{db} 7$ from the Daubechies family till fourth level decomposition. Garotte thresholding applied to detail coefficient D2, D3 and, D4 as shown in fig. 3. Fig. 4 shows the raw EMG signal and the denoised signal after the wavelet denoising.
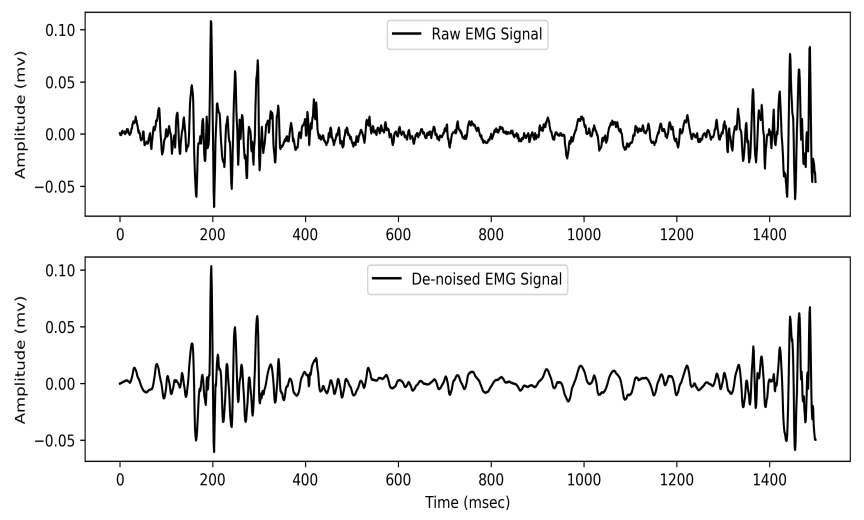

Fig. 4: Raw EMG signal and denoised EMG signal by using DWT

\section{B. Segmentation}

EMG signals are random. Due to its randomness, the segmentation of the EMG signal is necessary rather than the full signal. Windowing technique is being used for the segmentation of the signal. There are two different techniques (shown in fig 5) for data segmentation: overlapped windowing and adjacent windowing [20], [21]. In this paper, we considered overlapping windowing with $256 \mathrm{msec}$ window length and $25 \%$ overlapping [7].

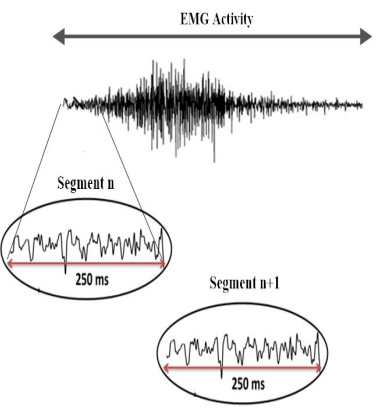

Adjacent Window

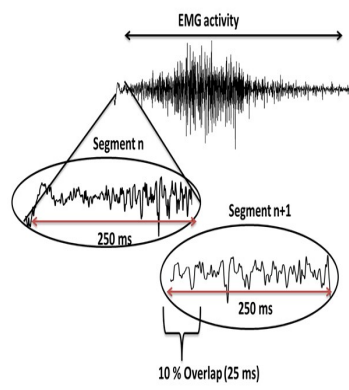

Overlapping Window
Fig. 5: Windowing Techniques

\section{Features Extraction and Selection}

Different types of artifacts and noises are still present after the preprocessing of the EMG data. These noises degrade the performance of the classification model. Different kinds of features (time domain, frequency domain, and time-frequency domain) are extracted from the pre-processed EMG data to improve the model performance. In this study, elven timedomain feature (shown in table I ) for four different muscle signals (i.e. 44 features) were being extracted for the knee abnormality classification.

After extracting the features, the selection of relevant features or remove the redundant features is a very challenging task. We applied the backward elimination procedure for the 
TABLE I: Mathematical expression of the extracted features

\begin{tabular}{|c|c|c|}
\hline Sr. No. & Feature Extracted & Mathematical Formulation \\
\hline 1 & Mean Absolute Value (MAV) & $\begin{array}{c}\frac{1}{N} \sum_{i=1}^{N}\left|x_{i}\right| \\
\text { Where }: x_{i} \text { is sample of EMG signal }\end{array}$ \\
\hline 2 & Root Mean Square (RMS) & $\sqrt{\frac{1}{N} \sum_{i=1}^{N}\left|x_{i}\right|^{2}}$ \\
\hline 3 & Zero Crossing (ZC) & 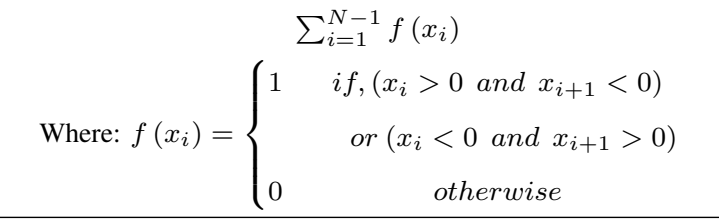 \\
\hline 4 & Slope Sign Change (SSC) & Where: $f\left(x_{i}\right)=\left\{\begin{array}{c}\sum_{i=2}^{N-1} f\left(x_{i}\right) \\
\text { if }, \text { if },\left(x_{i}>x_{i-1} \text { and } x_{i}>x_{i+1}\right) \\
\text { or }\left(x_{i}<x_{i-1} \text { and } x_{i}<x_{i+1}\right) \\
0\end{array}\right.$ \\
\hline 5 & Variance (VAR) & $\frac{1}{N-1} \sum_{i=1}^{N} x_{i}^{2}$ \\
\hline 6 & Wilison Amplitude (WAMP) & $\begin{array}{c}\sum_{i=1}^{N-1} f\left(\left|\left(x_{i+1}-x_{i}\right)\right|\right) \\
\text { Where: } f\left(x_{i}\right)=\left\{\begin{array}{lc}1 & \text { if },(x \geq \text { Threshold }) \\
0 & \text { otherwise }\end{array}\right.\end{array}$ \\
\hline 7 & Myopulse Percentage Rate (MYOP) & $\begin{aligned} & \frac{1}{N} \sum_{i=1}^{N} f\left(x_{i}\right) \\
& \text { Where: } f\left(x_{i}\right)=\left\{\begin{array}{cc}1 & \text { if },(x \geq \text { Threshold }) \\
0 & \text { otherwise }\end{array}\right.\end{aligned}$ \\
\hline 8 & Difference Absolute Standard Deviation Value (DASDV) & $\sqrt{\frac{1}{N-1} \sum_{i=1}^{N-1}\left(x_{i+1}-x_{i}\right)^{2}}$ \\
\hline 9 & Average Amplitude Change (AAC) & $\frac{1}{N} \sum_{i=1}^{N-1}\left|x_{i+1}-x_{i}\right|$ \\
\hline 10 & Skewness (Skew) & $\frac{E\left[(x-\mu)^{3}\right]}{\sigma^{3}}$ \\
\hline 11 & Kurtosis (Kurt) & $\begin{array}{l}\frac{E\left[(x-\mu)^{4}\right]}{\sigma^{4}} \\
\text { Where, } \sigma \text { is the Standard deviati of data set, } \\
\mu=\text { Mean of the data set, } \\
\text { E is the Expected value estimator of the signal }\end{array}$ \\
\hline
\end{tabular}

diagnosis of knee abnormality. In this approach we begin with all of the model's attributes, followed by their removal based on the p-value. Those attributes were discarded with p-values greater than 0.05 and the model was refitted with the rest of the attributes. This process was iterated several times until each existing variable was at a significant level for the model. After the selection of the feature, all selected features were standardized between 0 and 1 according to equation 2 .

$$
X_{F n e w}=\frac{X_{F}-X_{F \min }}{X_{F \max }-X_{F \min }}
$$

Where, $X_{\text {Fnew }}$ is the normalized EMG feature, $X_{F}$ is the actual EMG feature, $X_{F \max }$ is the maximum value of actual EMG feature, and $X_{F \min }$ is the minimum value of actual EMG feature.

\section{Machine Learning Methods}

In this section, discuss support vector machine, decision tree, k-nearest neighbor, random forest, and extra tree machine learning classifiers which were used for the classification between healthy and unhealthy individuals. We did not discuss the machine learning classifiers in depth.

Decision Tree (DT) [22] is a supervised learning classifier that can be used for both numerical or categorical data. It uses a tree kind of structure of decision-based on entropy.

- calculate the entropy of each features:

$$
H(X)=-\sum_{t \in Y} \boldsymbol{p}(t) \log _{2} p(t)
$$

Where, $\mathrm{X}$ is the Dataset, $\mathrm{Y}$ is the Set of classes in $\mathrm{S}$, and $\mathrm{p}(\mathrm{t})$ is number of elements in class $\mathrm{Y}$ to the number of elements in set $\mathrm{X}$

- Split the set into subsets using the attribute which information gain is maximum.

$$
I G(X, A)=H(X)-H(X \mid A)
$$


TABLE II: Performance Parameters of Different Classifiers

\begin{tabular}{|c|l|l|l|l|l|l|l|l|}
\hline \multirow{2}{*}{ Classifier } & \multicolumn{2}{|l|}{ Accuracy } & \multicolumn{2}{l|}{ Sensitivity } & \multicolumn{2}{l|}{ Specificity } & \multicolumn{2}{l|}{ F1-Score } \\
\cline { 2 - 9 } & $\mu$ & $\sigma$ & $\mu$ & $\sigma$ & $\mu$ & $\sigma$ & $\mu$ & $\sigma$ \\
\hline SVM & 0.701 & 0.019 & 0.404 & 0.028 & 0.864 & 0.024 & 0.550 & 0.027 \\
\hline DT & 0.700 & 0.018 & 0.567 & 0.043 & 0.773 & 0.022 & 0.653 & 0.028 \\
\hline KNN & 0.793 & 0.012 & 0.641 & 0.028 & 0.878 & 0.017 & 0.740 & 0.018 \\
\hline RF & 0.888 & 0.017 & 0.789 & 0.037 & 0.943 & 0.015 & 0.859 & 0.024 \\
\hline ET & 0.913 & 0.013 & 0.825 & 0.034 & 0.962 & 0.011 & 0.888 & 0.02 \\
\hline
\end{tabular}

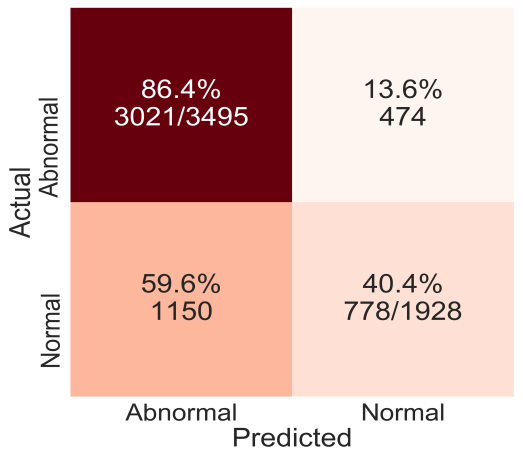

(a) SVM

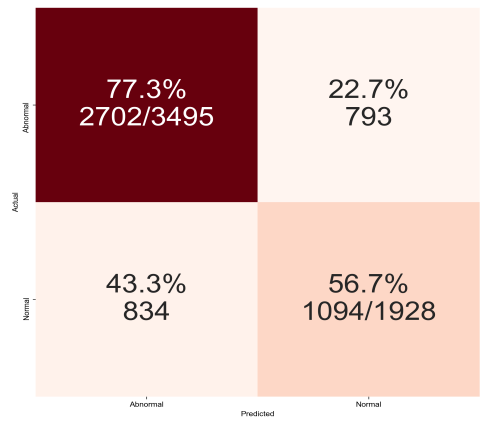

(b) Decision Tree

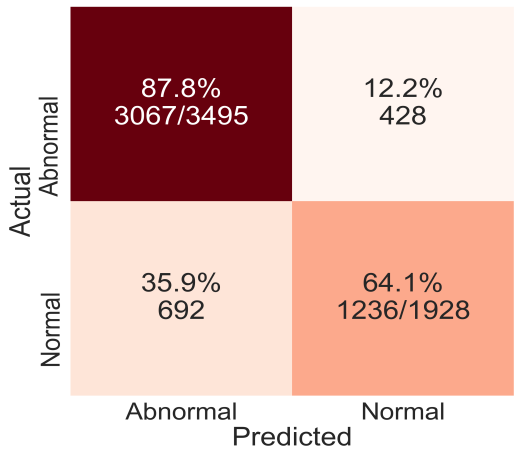

(c) $\mathrm{KNN}$

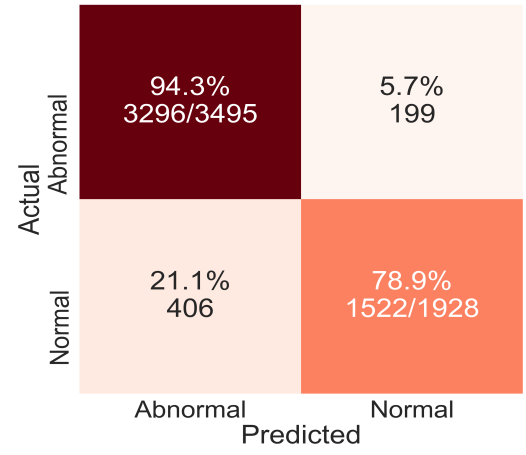

(d) Random Forest

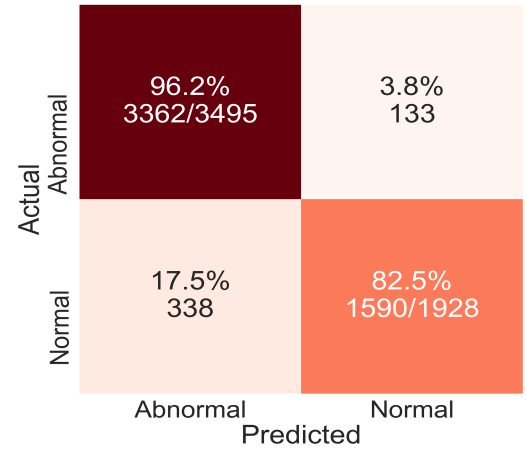

(e) Extra Tree

Fig. 6: Confusion Matix of Different Classifiers

- No further splitting is required if the value of entropy is 0 , otherwise further splitting the same as above.

Random Forest (RF) [23] is a similar kind of decision tree algorithm but it creates several trees rather than a single tree.

- First, select the number of samples in the dataset randomly which is called bootstraps sample.

- Create the decision tree for every bootstrap sample and calculate the prediction results from each decision tree.

- Voting was performed between the results of decision trees, and the most voted prediction result shows the output of the overall prediction.
Extra Tree (ET) [24] is very similar to the random forest classifier. It considers the entire dataset instead of the bootstrap dataset.

\section{RESUlt AND Discussion}

This section presents the performance analysis of different machine learning classifiers. This problem is a binary class problem either subject is healthy or unhealthy. A machine learning algorithm for binary classification gives four outcomes which are: True Negative (TN), True Positive (TP), False Negative (FN), and False Positive (FP). Calculate the performance parameters with the help of these four parameters. 


$$
\begin{gathered}
\text { Accuracy }=\frac{T P+T N}{T P+T N+F P+F N} \\
\text { Precision }=\frac{T P}{T P+F P} \\
\text { Sensitivity }(\text { Recall })=\frac{T P}{T P+F N} \\
\text { Specificity }=\frac{T N}{T N+F P} \\
F 1-\text { Score }=\frac{2 * \text { Recall } * \text { Precision }}{\text { Recall }+ \text { Precision }}
\end{gathered}
$$

$\mathrm{K}$-fold cross-validation is a re-sampling method that used a constrained data test to evaluate the performance parameters of machine learning models. In this k-fold approach, data are randomly separated into $\mathrm{k}$ groups of equal size. Then the model is trained with the help of k-1 groups of a dataset and validates with $k^{t h}$ dataset. This process is repeated for all the groups of a dataset. In this article, performance parameters of classification models were evaluated by using 10 fold crossvalidation which is shown in table II. The accuracy for the extra tree classifier was $91.3 \%$ while it was $70.1 \%, 70.0 \%$, $79.3 \%$, and $88.8 \%$ for the support vector machine, decision tree, k-nearest neighbor and random forest respectively. Extra Tree classifier shows high-performance parameters than other machine-learning classifiers. Fig. 6 shows the confusion matrix for the knee abnormality classification.

\section{Conclusion and Future Scope}

Comparative analysis of machine learning classifiers for sEMG based classification of lower limb abnormality in between subjects suffering from knee abnormality and healthy subjects have been studied. First, raw EMG signal denoised with the help of a discrete wavelet transform then 11 handcrafted features were extracted by using overlapping windowing techniques. After that relevant features were selected by using the backward selection method. Then, five different machine learning models are being used to calculate the performance parameter in which extra tree classifier reflects superlative performance than other classifiers. In this study, publicly available data taken from the UCI. only data for 22 subjects are available. As a future scope, we can collect EMG data for more number of subjects.

\section{REFERENCES}

[1] Daniel K White, Catrine Tudor-Locke, David T Felson, K Douglas Gross, Jingbo Niu, Michael Nevitt, Cora E Lewis, James Torner, and Tuhina Neogi. Do radiographic disease and pain account for why people with or at high risk of knee osteoarthritis do not meet physical activity guidelines? Arthritis \& Rheumatism, 65(1):139-147, 2013.

[2] J Bedson, K Jordan, and P Croft. How do gps use $\mathrm{x}$ rays to manage chronic knee pain in the elderly? a case study. Annals of the rheumatic diseases, 62(5):450-454, 2003.

[3] Charles Peterfy and Manish Kothari. Imaging osteoarthritis: magnetic resonance imaging versus X-ray. Current rheumatology reports, 8(1):16, 2006.
[4] Okan Erkaymaz, Şenyer İrem, and Rukiye Uzun. Detection of knee abnormality from surface emg signals by artificial neural networks. In 2017 25th Signal Processing and Communications Applications Conference (SIU), pages 1-4. IEEE, 2017.

[5] Tahir Hussain, Hafiz Farhan Maqbool, Nadeem Iqbal, Mukhtaj Khan, Salman, and Abbas A Dehghani-Sanij. Computational model for the recognition of lower limb movement using wearable gyroscope sensor. International Journal of Sensor Networks, 30(1):35-45, 2019.

[6] Orkun Tunçel, Kerem Altun, and Billur Barshan. Classifying human leg motions with uniaxial piezoelectric gyroscopes. Sensors, 9(11):8508$8546,2009$.

[7] Ganesh R Naik, S Easter Selvan, Sridhar P Arjunan, Amit Acharyya, Dinesh K Kumar, Arvind Ramanujam, and Hung T Nguyen. An icaebm-based semg classifier for recognizing lower limb movements in individuals with and without knee pathology. IEEE Transactions on Neural Systems and Rehabilitation Engineering, 26(3):675-686, 2018.

[8] Mario Nieto-Hidalgo, Francisco Javier Ferrández-Pastor, Rafael J Valdivieso-Sarabia, Jerónimo Mora-Pascual, and Juan Manuel GarcíaChamizo. A vision based proposal for classification of normal and abnormal gait using rgb camera. Journal of biomedical informatics, 63:82-89, 2016

[9] He Zhang and Cang Ye. Rgb-d camera based walking pattern recognition by support vector machines for a smart rollator. International journal of intelligent robotics and applications, 1(1):32-42, 2017.

[10] Dario Farina and Francesco Negro. Accessing the neural drive to muscle and translation to neurorehabilitation technologies. IEEE Reviews in biomedical engineering, 5:3-14, 2012.

[11] Ankit Vijayvargiya, Puru Lokendra Singh, Samidha Mridul Verma, Rajesh Kumar, and Sanjiv Bansal. Performance comparison analysis of different classifier for early detection of knee osteoarthritis. In Sensors for Health Monitoring, pages 243-257. Elsevier, 2019.

[12] Ömer Faruk Ertuğrul, Yılmaz Kaya, and Ramazan Tekin. A novel approach for semg signal classification with adaptive local binary patterns. Medical \& biological engineering \& computing, 54(7):11371146, 2016.

[13] OFA Sanchez, JLR Sotelo, MH Gonzales, and GAM Hernandez. Emg dataset in lower limb data set. UCI machine learning repository, pages 2014-02, 2014

[14] Ching-Fen Jiang and Shou-Long Kuo. A comparative study of wavelet denoising of surface electromyographic signals. In 2007 29th Annual International Conference of the IEEE Engineering in Medicine and Biology Society, pages 1868-1871. IEEE, 2007.

[15] Adriano O Andrade, Slawomir Nasuto, Peter Kyberd, Catherine M Sweeney-Reed, and FR Van Kanijn. Emg signal filtering based on empirical mode decomposition. Biomedical Signal Processing and Control, 1(1):44-55, 2006.

[16] Alexandros Karagiannis and Philip Constantinou. Noise-assisted data processing with empirical mode decomposition in biomedical signals. IEEE Transactions on Information Technology in Biomedicine, 15(1):11-18, 2010

[17] M Kania, M Fereniec, and R Maniewski. Wavelet denoising for multilead high resolution ecg signals. Measurement science review, 7(4):3033, 2007.

[18] Zhi-dong Zhao, Min Pan, Guang Li, and Yu-quan CHEN. Independent component analysis (ica) for denoising emg signal from ecg signal. JOURNAL-ZHEJIANG UNIVERSITY ENGINEERING SCIENCE, 38(1):103-107, 2004.

[19] Clemens Valens. A really friendly guide to wavelets. ed. Clemens Valens, 1999.

[20] Nurhazimah Nazmi, Abdul Rahman, Mohd Azizi, Shin-Ichiroh Yamamoto, Siti Anom Ahmad, Hairi Zamzuri, and Saiful Amri Mazlan. A review of classification techniques of emg signals during isotonic and isometric contractions. Sensors, 16(8):1304, 2016.

[21] Mohammadreza Asghari Oskoei and Huosheng Hu. Support vector machine-based classification scheme for myoelectric control applied to upper limb. IEEE transactions on biomedical engineering, 55(8):19561965, 2008.

[22] J. Ross Quinlan. Induction of decision trees. Machine learning, 1(1):81$106,1986$.

[23] Leo Breiman. Random forests. Machine learning, 45(1):5-32, 2001.

[24] Pierre Geurts, Damien Ernst, and Louis Wehenkel. Extremely randomized trees. Machine learning, 63(1):3-42, 2006. 